\title{
Autoimmune polyendocrinopathy-candidiasis-ectodermal dystrophy syndrome (APECED) due to AIRET16M mutation in a consanguineous Greek girl
}

\author{
Konstantinos Kollios ${ }^{1, a}$, Anastasia Tsolaki ${ }^{1, a}$, \\ Charalampos Antachopoulos ${ }^{1}$, I. Moix ${ }^{2}$, Michael \\ A. Morris ${ }^{2}$, Maria Papadopoulou ${ }^{1}$ and Emmanuel \\ Roilides $^{1, *}$ \\ ${ }^{1}$ Third Department of Pediatrics, Aristotle University \\ School of Medicine, Hippokration Hospital, Thessaloniki, \\ Greece \\ ${ }^{2}$ Molecular Diagnostic Laboratory, Geneva University \\ Hospitals, Geneva, Switzerland
}

\begin{abstract}
Autoimmune polyendocrinopathy-candidiasis-ectodermal dystrophy syndrome (APECED) or autoimmune polyendocrine syndrome type 1 (APS-1) is a rare autosomal recessive disease caused by mutations of the AutoImmune REgulator (AIRE) gene, an important mediator of tolerance to self-antigens. It is characterized by two out of three major components: chronic mucocutaneous candidiasis, hypoparathyroidism and Addison's disease. We present an 11-yearold girl suffering from recurrent episodes of mucocutaneous candidiasis and onychomycosis from 1 to 6 years of age, and transient alopecia at the age of 4 years. Hypoparathyroidism and dental enamel hypoplasia were diagnosed at 8 years. Autoantibodies to thyroid and adrenal glands were not detected and all other endocrine functions have remained normal. Genetic analysis revealed that the patient was homozygous for the mutation T16M in exon 1 of the AIRE gene (p.T16M, c. $47 \mathrm{C}>\mathrm{T}$ ). This is the first APECED case reported for carrying this mutation in homozygous form. Parents were third cousins and heterozygous carriers of this mutation.
\end{abstract}

Keywords: autoimmune polyendocrine syndrome type 1 (APS-1); AutoImmune REgulator gene; chronic mucocutaneous candidiasis; consanguinity; Greece; hyperphosphatemia; hypoparathyroidism.

\footnotetext{
${ }^{a}$ The two authors equally contributed to the care of the patient and to the writing of this report.

*Corresponding author: Emmanuel Roilides, 3rd Department of Pediatrics, Aristotle University School of Medicine, Hippokration Hospital, Konstantinoupoleos 49, GR-546 42 Thessaloniki, Greece Phone: +30-2310-892444, Fax: +30-2310-992981,

E-mail: roilides@med.auth.gr
}

\section{Introduction}

Autoimmune polyendocrinopathy-candidiasis-ectodermal dystrophy syndrome (APECED) or autoimmune polyendocrine syndrome type 1 (APS-1) is a rare autosomal recessive disease (1-3) generally seen in infants and children that is caused by mutations of the AutoImmune REgulator (AIRE) gene on chromosome 21q22.3 $(4,5)$. It is characterized by the presence of at least two of three components: chronic mucocutaneous candidiasis, hypoparathyroidism and Addison's disease, but can also include other clinical manifestations, such as autoimmune thyroid disease, type 1 diabetes mellitus, alopecia, vitiligo, ectodermal dystrophy, malabsorption and pernicious anemia (6).

The aim of this report is to present the first genetically confirmed case of APECED in Greece (the fourth worldwide according to PubMed), in which the syndrome was found to be due to homozygosity for a very rare mutation of AIRE, p.T16M.

\section{Case report}

The patient, now an 11-year-old girl, was first referred to the Infectious Diseases Clinic of our Department at the age of 3 years due to frequent relapses of mucocutaneous candidiasis. She was the second child of reportedly non-consanguineous parents from the South-eastern Aegean island of Rhodes, Greece. Clinical examination on admission revealed candidiasis of the oral cavity, axillary folds, and nails of both hands. Mucocutaneous candidiasis had appeared at the age of 1 year and the patient kept relapsing until the age of six. During the first 5 years the frequency of the episodes was once a month, whereas during the 6th year they occurred every 3 months. Multiple cultures from the oral cavity grew Candida albicans. Candidiasis of the axillary folds lasted for 1 month and onychomycosis for 6 months. At the age of 4 years the patient also presented with transient alopecia and hair-thinning that lasted for about 4 months. Each relapse of mucocutaneous candidiasis was effectively treated with oral itraconazole solution $(5 \mathrm{mg} / \mathrm{kg} / \mathrm{day})$. The patient was followed up at the Infectious Diseases Clinic of our Department of Pediatrics annually.

Immunological tests at the age of 3 years, including immunoglobulins and cell-mediated immune response, were normal. Three years later, follow-up revealed relatively low levels of $\mathrm{IgM}$, increased levels of $\mathrm{C} 3$ and low rhodamine oxidation (oxidative burst) of phagocytes. Antinuclear antibodies (ANA) and anti-neutrophil cytoplasmic (ANCA) antibodies were negative. After the age of 6 years, repetitive cultures 
from pharynx and oral cavity during the annual visits were negative, while biochemical tests were all normal.

During a follow-up visit at the age of 8 years, laboratory tests revealed hypoparathyroidism suggested by low serum calcium $(\mathrm{Ca})$ and high phosphate $(\mathrm{P})$ levels $(7.1$ and $8.9 \mathrm{mg} / \mathrm{dL}$, respectively), and intact parathormone $3 \mathrm{pg} / \mathrm{mL}$ (normal levels $>7 \mathrm{pg} / \mathrm{mL}$ ). As the patient fulfilled two major diagnostic criteria for the diagnosis of APECED, additional investigations were performed in order to exclude other conditions possibly associated with this syndrome. Serum levels of cortisol before and after administration of adrenocorticotropic hormone (ACTH) were normal. Autoantibodies against several organs [ANA, antithyroid antibodies (ATA), anti-DNA, smooth muscle antibodies (SMA), parietal cells antibodies (PCA), liver/ kidney/microsomal antibodies (LKM), islet cell antibodies (ICA)] were negative. A dental exam at this age revealed dental enamel hypoplasia.

Hypoparathyroidism was initially treated with calcitriol and calcium carbonate. With this treatment, calcium levels were completely restored. Later, low phosphorus diet and the non-calcium based binder sevelamer were added. During follow-up, renal function remained normal. In addition, there was no calciuria or proteinuria (evidenced as urinary excretion of albumin and $\beta_{2}$ microglobulin), and there was no evidence of nephrocalcinosis in renal ultrasound.

\section{Genetic analysis}

The diagnosis of APECED was genetically confirmed at the age of 10 years, in the Molecular Diagnostic Laboratory, Geneva University Hospitals. Polymerase chain reaction amplification from leukocyte DNA followed by DNA sequencing of the entire AIRE coding region (primer sequences available on request) revealed that the patient was homozygous for the mutation T16M in exon 1 of the AIRE gene (p.T16M, c.47C>T). Both parents were found to be heterozygous carriers of the mutation. Despite their insistent denial of consanguinity, a thorough investigation revealed that the parents were third cousins.

The patient's 12-year-old brother was asymptomatic without any abnormal signs on physical examination. His serum levels of $\mathrm{Ca}$ and $\mathrm{P}$ were normal. According to the wish of his parents, no further investigations were performed.

\section{Discussion}

APECED is a rare disease, but is more frequent in certain populations. Its prevalence is estimated to be $1: 80,000$ in Norway, 1:30,000 in Ireland, 1:25,000 in Finland, 1:14,000 in Sardinia, Italy and 1:9000 among Iranian Jews (7). The largest reported series was conducted in Finland in 2006 and included 91 patients (2).

APECED has three major clinical components: chronic mucocutaneous candidiasis, hypoparathyroidism, and Addison's disease. The presence of at least two of them is required for the clinical diagnosis (7). Although this diagnostic criterion is valid, it leaves many patients unrecognized. Recently,
Meloni et al. proposed the presence of autoantibodies against interferon $-\omega$ and $\alpha_{2}$ to diagnose APECED in patients with just one of the APECED triad $(7,8)$. Due to its high sensitivity and specificity this test has been proposed as a simpler diagnostic option than detecting AIRE mutations (8).

APECED is caused by mutations of the AIRE gene, which consists of 14 exons and is located on chromosome $21 \mathrm{q} 22.3$ (2). To date, about 60 mutations have been reported, and comprise nonsense and missense mutations, deletions, and small insertions (9). The mutations R257X (exon 6), c.967-979del13 (exon 8), R139X (exon 3), and Y85C (exon 2) appear to be more common (10). The most common "Finnish" mutation $\mathrm{R} 257 \mathrm{X}$ results in a premature stop codon in the SAND domain of AIRE protein $(2,10)$. The mutation of our patient's AIRE T16M is very rare and, to the best of our knowledge, has been reported only in three patients (one Russian and two Slovenians) to date (Table 1). All four patients suffered from mucocutaneous candidiasis and hypoparathyroidism, whereas three of the three patients with data available suffered from ectodermal dystrophy. By comparison, only one patient each suffered from hypothyroidism or Addison's disease.

Our patient is the first known patient homozygous for this mutation, confirming its identity as a classic APECED mutation and adding APECED to the list of very rare recessive disorders to be identified in consanguineous couples (the probability of third cousins to share a specific allele is $1 / 128)$. The denial of consanguinity by the couple underlines the difficulties for individuals and professionals alike to develop accurate and comprehensive family histories. It will be interesting to search for further cases of patients with T16M from the same region.

The AIRE protein contains several structural motifs common to nuclear proteins and transcription factors (11). AIRE gene is predominantly expressed within the thymus in a specialized subset of thymic stromal cells known as medullary thymic epithelial cells and controls ectopic expression of tissue-restricted antigens (12). AIRE-mediated regulation of

Table 1 Reported cases with AIRE gene mutation T16M.

\begin{tabular}{lllll}
\hline Patient & $\# 1$ & $\# 2$ & $\# 3$ & Present \\
\hline Year of publication & 2001 & 2005 & 2005 & 2011 \\
(Ref) & $(4)$ & $(5)$ & $(5)$ & \\
Age at first clinical & NA & 3.7 years & 5.5 years & 1 year \\
manifestations & & & & \\
Ethnicity & Russian & Slovenian & Slovenian & Greek \\
Sex (M/F) & NA & M & F & F \\
AIRE mutation & R257X/ & R257X/ & R257X/ & T16M/ \\
& T16M & T16M & T16M & T16M \\
Clinical characteristics & & & & \\
MC & + & + & + & + \\
HP & + & + & + & + \\
AD & + & - & - & - \\
ED & NA & + & + & + \\
HT & + & - & - & - \\
MA & NA & + & - & - \\
A & NA & + & - & + \\
\hline
\end{tabular}

A, alopecia; AD, Addison's disease; ED, ectodermal dystrophy; HP, hypoparathyroidism; HT, hypothyroidism; MA, malabsorption; MC, mucocutaneous candidiasis; NA, not available. 
diverse self-antigens in the thymus serves as a paradigm for the importance of promiscuous gene expression in the prevention of autoimmune disease (11).

Our immunological findings of constantly low levels of IgM, increased levels of $\mathrm{C} 3$ and low oxidative bursts of phagocytes appear to fit in the general immunological scenario of APECED, in which a number of different disturbances of immune functions have been found, such as complement dysfunction, immunoglobulin deficiencies and innate immune response disturbances (e.g., impaired dendritic cell maturation, but not impaired pattern recognition receptors) $(13,14)$.

A high clinical suspicion should be maintained for timely detection of other autoimmune disorders in patients with APECED and their close relatives, especially siblings. Regular follow-up is very important for the prevention of serious sequelae of the disease.

As this is the first genetically confirmed case in Greece, it is important to note that the clinical and genetic findings are consistent with most cases of APECED reported in the literature. This similarity exists despite the fact that our patient is only the fourth found having AIRE T16M, and the first to be homozygous. Close follow-up of endocrine and renal function is warranted in such a rare case.

\section{Acknowledgments}

We thank the pediatrician of the patient, Dr Nikos Chatzinikolas, who referred her to our department and for his continuous excellent co-operation during the care of the patient.

\section{Conflict of interest statement}

Authors' conflict of interest disclosure: The authors have no relevant financial interests in this manuscript.

\section{References}

1. Ahonen P. Autoimmune polyendocrinopathy-candidosis-ectodermal dystrophy (APECED): autosomal recessive inheritance. Clin Genet 1985;27:535-42.
2. Perheentupa J. Autoimmune polyendocrinopathy-candidiasisectodermal dystrophy. J Clin Endocrinol Metab 2006;91: 2843-50.

3. Jaaskelainen J, Perheentupa J. Autoimmune polyendocrinopathy-candidosis-ectodermal dystrophy (APECED) - a diagnostic and therapeutic challenge. Pediatr Endocrinol Rev 2009;7: 95-108.

4. Cihakova D, Trebusak K, Heino M, Fadeyev V, Tiulpakov A, et al. Novel AIRE mutations and P450 cytochrome autoantibodies in Central and Eastern European patients with APECED. Hum Mutat 2001;18:225-32.

5. Podkrajsek KT, Bratanic N, Krzisnik C, Battelino T. Autoimmune regulator-1 messenger ribonucleic acid analysis in a novel intronic mutation and two additional novel AIRE gene mutations in a cohort of autoimmune polyendocrinopathy-candidiasisectodermal dystrophy patients. J Clin Endocrinol Metab 2005; 90:4930-5.

6. Betterle C, Zanchetta R. Update on autoimmune polyendocrine syndromes (APS). Acta Biomed 2003;74:9-33.

7. Husebye ES, Perheentupa J, Rautemaa R, Kampe O. Clinical manifestations and management of patients with autoimmune polyendocrine syndrome type I. J Intern Med 2009;265:514-29.

8. Meloni A, Furcas M, Cetani F, Marcocci C, Falorni A, et al. Autoantibodies against type I interferons as an additional diagnostic criterion for autoimmune polyendocrine syndrome type I. J Clin Endocrinol Metab 2008;93:4389-97.

9. Kahaly GJ. Polyglandular autoimmune syndromes. Eur J Endocrinol 2009;161:11-20.

10. Heino M, Scott HS, Chen Q, Peterson P, Maebpaa U, et al. Mutation analyses of North American APS-1 patients. Hum Mutat 1999;13:69-74.

11. Cheng MH, Shum AK, Anderson MS. What's new in the AIRE? Trends Immunol 2007;28:321-7.

12. Peterson P, Org T, Rebane A. Transcriptional regulation by AIRE: molecular mechanisms of central tolerance. Nat Rev Immunol 2008;8:948-57.

13. Ryan KR, Hong M, Arkwright PD, Gennery AR, Costigan C, et al. Impaired dendritic cell maturation and cytokine production in patients with chronic mucocutanous candidiasis with or without APECED. Clin Exp Immunol 2008;154:406-14.

14. Hong M, Ryan KR, Arkwright PD, Gennery AR, Costigan C, et al. Pattern recognition receptor expression is not impaired in patients with chronic mucocutanous candidiasis with or without autoimmune polyendocrinopathy candidiasis ectodermal dystrophy. Clin Exp Immunol 2009;156:40-51. 